\title{
Telehealth Using PoseNet-Based System for In-Home Rehabilitation
}

\author{
Jiaming Chua (D), Lee-Yeng Ong *(D) and Meng-Chew Leow (D) \\ Faculty of Information Science and Technology, Multimedia University, Jalan Ayer Keroh Lama, \\ Melaka 75450, Malaysia; 1171101251@student.mmu.edu.my (J.C.); mcleow@mmu.edu.my (M.-C.L.) \\ * Correspondence: lyong@mmu.edu.my; Tel.: +60-6-2523761
}

Citation: Chua, J.; Ong, L.-Y.; Leow, M.-C. Telehealth Using PoseNet-Based System for In-Home Rehabilitation. Future Internet 2021, 13, 173. https://doi.org/ 10.3390/ fi13070173

Academic Editors:

Matthew Pediaditis and Joel José Puga Coelho Rodrigues

Received: 12 May 2021

Accepted: 24 June 2021

Published: 2 July 2021

Publisher's Note: MDPI stays neutral with regard to jurisdictional claims in published maps and institutional affiliations.

Copyright: (C) 2021 by the authors Licensee MDPI, Basel, Switzerland. This article is an open access article distributed under the terms and conditions of the Creative Commons Attribution (CC BY) license (https:/ / creativecommons.org/licenses/by/ $4.0 /)$.

\begin{abstract}
The increasing cost of healthcare services is accelerating the development of the telehealth system to fulfill the necessity of delivering an efficient and cost-effective remote healthcare services. Moreover, the ageing of the global population and the disruption of the COVID-19 pandemic are creating a rapid rise of demand for healthcare services. This includes those who are in need of remote monitoring for chronic conditions through rehabilitation exercises. Therefore, this paper presents a telehealth system using PoseNet for in-home rehabilitation, with built-in statistical computation for doctors to analyze the patient's recovery status. This system enables patients to perform rehabilitation exercises at home using an ordinary webcam. The PoseNet skeleton-tracking method is applied to detect and track the patients' angular movements for both elbows and knees. By using this system, the measurement of the elbow and knee joint angles can be calculated and recorded while patients are performing rehabilitation exercises in front of the laptop webcam. After the patients complete their rehabilitation exercises, the skeleton results of four body parts will be generated. Based on the same actions performed by patients on selected days, the doctors can examine and evaluate the deviation rate of patients' angular movements between different days to determine the recovery rate.
\end{abstract}

Keywords: telehealth; PoseNet; in-home rehabilitation; angular movement; skeleton result

\section{Introduction}

Telehealth refers to the delivery of health-related services including medical care, health education and health information services over a distance via the use of digital information and communication technologies such as computers, laptops and mobile devices [1]. Telehealth was created to overcome the physical barriers in offering healthcare services to patients and caregivers without in-person visits and to reduce the travelling challenges for patients in rural areas [2]. On top of this, rapid ageing of the global population has brought unprecedented and escalating challenges to the healthcare services. According to data provided by the World Health Organization (WHO), the proportion of the global population over the age of 60 will rise from $12 \%$ to a staggering $22 \%$, almost doubling from 2015 to 2050 [3]. These elderly, especially those with chronic conditions, are not fit to travel long distance for healthcare services. Therefore, telehealth plays a crucial role in the seamless delivery of an efficient and cost-effective remote healthcare services for these people. In addition, the current COVID-19 pandemic has also induced the rapid adoption of telehealth and instigated adaptation and reform in the delivery of healthcare services [2]. Studies have shown that $90 \%$ of patients and parents felt safer using a telehealth virtual appointment compared to an in-person visit during the COVID-19 pandemic [4]. The functionality and scope of the telehealth system are rapidly increasing and well received by patients and clinicians.

By leveraging the advantage of telehealth to facilitate remote healthcare services, in-home rehabilitation offers diverse healthcare services to individual households. This covers a range of activities, from assessing the patient's functional ability in his/her environment to specific supervised treatment or exercises [5]. In most countries nowadays, in-home rehabilitation services are strongly supported by the government. The number of 
patients applying for such services has grown significantly, adopting family assessment and occupational therapy to enhance the performance of daily activities [6]. It can be effectively used in physical therapy, such as postoperative knee rehabilitation $[7,8]$. As a result, in-home rehabilitation offers a lot of benefits for patients via the use of a telehealth system.

Traditionally, patients perform rehabilitation exercises in hospitals or rehabilitation centers. In Malaysia, rehabilitation services are offered by both governmental and nongovernmental agencies [9]. Some challenges in these provisions include the lack of coordination among these agencies and severe service barriers for patients in rural areas with poor transportation, or for whom driving is unsuitable because of their medical condition. One way to address this issue is to hire a home therapist who visits the patient's house, but not all patients can afford the higher cost. An alternative solution is to offer in-home rehabilitation services via the use of a telehealth system.

Previously, a marker-based system was used for in-home rehabilitation to detect patient's movements by placing markers on their bodies. However, the cost of these marker-based systems is high [10]. An example of a low-cost marker-based system such as Perceptron Neuron is priced at USD\$1499 (approximately RM 6000) in Malaysia [11]. According to the Malaysian Department of Statistics, the average monthly household income of the bottom $40 \%$ of the population (the B40 group) is below RM 4849, while the average monthly household income of the middle $40 \%$ of the population (the M40 group) ranges from RM 4850 to RM 10,959 [12]. Hence, Perceptron Neuron is obviously beyond the reach of the B40 and M40 household income groups in Malaysia. On top of this, professional skill is required to place the markers correctly on the bodies [13]. If the markers are misplaced, it might lead to an inaccurate detection. In addition, it is also a timeconsuming process and patients might experience some discomfort when many markers are attached to the body for long hours during the rehabilitation procedure [13]. Due to the high cost of the marker-based system, a more affordable system using Microsoft Kinect was introduced in 2010 [10,14-23]. Most importantly, this system eliminates the need to place markers on the body. After the discontinuation of Microsoft Kinect in the fall of 2017, another skeleton recognition technology, the PoseNet, which uses TensorFlow.js library, emerged for movement detection [15]. Unlike Microsoft Kinect, patients are not required to purchase the sensor or attach markers to their body. PoseNet can detect patient's movement with just an ordinary webcam attached to the laptop.

In this paper, a PoseNet-based telehealth system for in-home rehabilitation is introduced. The system produces a set of statistical computations based on the patient's angular movements for comparison and evaluation by the doctors. This enables doctors to conduct in-depth analysis. On top of this, the system offers the potential to fill in the gaps by: (1) using an ordinary webcam with internet connection for rehabilitation exercises; (2) providing access for both patient and doctor to the skeleton results in a timely manner as soon as the patient has completed the rehabilitation exercises; and (3) displaying the percentage of deviation rate of the patient's skeleton results for different days, for the doctor to further analyze the patient's recovery status. Our aim is to assist the patient in performing rehabilitation exercises at home without increasing their financial burden and travelling challenges. This is especially useful for elderly patients with limited mobility.

During in-home rehabilitation, the patient needs to stand in front of the laptop webcam to perform the rehabilitation exercises. PoseNet will detect the posture and record the angular movements of both elbows and knees. The patient can obtain the skeleton data after completing the rehabilitation exercises for evaluation. The patient needs to continue with the same rehabilitation exercises for the selected number of days so that the doctors can monitor their rehabilitation postures and examine the deviation rate of the patient's angular movements to determine their recovery rate. They can then receive advice or recommendations from the doctors. As a result, the patient can improve their postural movements based on the doctor's prescriptions. Hence, it is possible to implement an in-home rehabilitation procedure using the PoseNet-based telehealth system, especially for low-income patients in Malaysia. 
The rest of the paper is organized as follows. First, Section 2 reviews some of the related studies that deliver similar systems. Next, the proposed telehealth system is elaborated in Section 3. The methodology for the functionality test is presented in Section 4. Section 5 clarifies the discussions of the results obtained. Finally, the conclusion is discussed in the last section.

\section{Related Work}

Various rehabilitation systems or applications have been currently developed, such as the virtual reality and motion-based games [16]. As a result, these applications have attracted great attention and interest among researchers. These applications motivate patients to move their body parts more frequently and offer patients a sense of accomplishment through an interactive user interface. Patients will need to follow the step-by-step rehabilitation exercises as guided by a 3D avatar or by following the recorded instructional video by the professional physician.

Anton et al., Yu et al., Chiang et al., Lin et al. and Su et al. [16-20] presented a Kinectbased rehabilitation system by integrating different approaches. Research in [16-18] focuses on integrating a 3D avatar for rehabilitation exercises. The system matches the patients' movements with the benchmark movements of a 3D avatar to determine the accuracy of the posture. The authors in [16] determine the accuracy of the posture by measuring the differences between the 3D angle of the joints and limbs. After the comparison, the system will display five different messages, namely "Execute the movement (has reached the initial position and has to execute the movement)", "Execute posture (very far from reaching the next posture)", "Cool, cool (about to reach the position)", "You're close (very close to the position)", "Correct (posture is correct)". The authors of $[17,18]$ adopt the dynamic time warping (DTW) approach to calculate the optimal warping distance in order to measure the similarity between two motion sequences. The optimal warping distance is then converted to a performance score (0-100\%) to show the similarity between the patient's and the 3D avatar's movement.

Research in $[19,20]$ adopts a recorded instructional video to guide patients to perform rehabilitation exercises at home. The patients need to follow the exercises as guided by a physician in the recorded instructional video. After completing the rehabilitation exercises, joint distance is calculated in [19] based on the captured patient's skeleton keypoints and the law of cosine is applied to calculate the angle differences between patients' movement and the standard movement of the physician. A quantitative score is then provided by dividing the score into four levels of comments, namely "Try it again", "Good Work", "Excellent" and "Perfect". On the contrary, the research in [20] integrated DTW with Euclidean distance and fuzzy logic to convert the optimal warping distance to three performance levels, namely "Bad", "Good" and "Excellent", to determine the accuracy of the posture.

Four sets of the Kinect-based rehabilitation system were presented in [21-24]. These research papers were published based on a project that was designed to focus on patients who usually suffer from movement disorders and neurological disorders that require in-home rehabilitation. The system in [21] presents a virtual trainer for patients to imitate movements during the rehabilitation exercises. Based on the 2D keypoints obtained from the hands and legs of patients, the joint angle is calculated by using the law of cosine. The calculated joint angle is then used to compare with the benchmark joint angle of the virtual trainer to evaluate the deviation of the patients' movement angle. On the other hand, researchers in [22-24] designed a video game for rehabilitation exercises with the aim of increasing the motivation of patients to perform in-home rehabilitation. These three papers offer several rehabilitation games that require the use of virtual movements to control the games. The patients need to move their bodies and perform a series of specific movements to achieve the goal. This is also beneficial for patients because they exercise their bodies more often while playing. Researchers in [22,23] implement games based on different difficulty levels to monitor patients' progress. The paper in [24] measured and recorded 
the patients' angular movement during game play. After the patients have completed the games, an angular movement graph will be generated, enabling the physiotherapist to monitor whether the patients achieve the accuracy of the required movement.

The aforementioned papers use Kinect sensors as the input device to detect the skeleton movements of the patients in their rehabilitation systems. The patients need to purchase a Kinect sensor to use these systems. Alternatively, Adolf et al. [25] developed a rehabilitation system, OpenPose, which uses a computer vision approach to detect the keypoints and skeleton movements of the patients. This has created an opportunity for in-home rehabilitation system using only a laptop or mobile camera, eliminating the need to purchase any additional device or sensor. When the patients perform rehabilitation exercises, they only need to stand in front of their laptop or mobile camera to allow OpenPose to detect their skeleton movements. After the patients have completed the rehabilitation exercises, the angles are computed between the body parts using the captured data of the patients' skeleton keypoints. An angular movement graph will be generated for the physiotherapist to perform manual assessment by comparing with the angular movements performed by the professional trainer to monitor the accuracy of the patients movements. Despite its lower cost, manually examining the patients' posture through an angular movements graph may be tedious and susceptible to fatigue error.

On the other hand, Hassan et al. [26] proposed a PoseNet-based rehabilitation system to assist patients who undergo physiotherapy exercises. This system utilizes the PoseNet to detect and extract the skeleton keypoints of patients from the 30-s live exercise video. A 3D avatar performs the exercise to guide the patient in the correct postures. After recording, the PoseNet model will extract the patient's skeleton keypoints. The score is then calculated by using the DTW approach to measure the similarity between the keypoints of the patients and the benchmark keypoints performed by the experts. Finally, the patient's score $(0-100 \%)$ will be provided based on all the exercise movements along with a short feedback statement to advice on incorrect performance, such as, "Work on your left elbow", "Work on your right wrist" and "Good job".

In summary, the existing in-home rehabilitation systems come with a few problems as follows: (1) A special-purpose hardware such as Kinect is required; (2) There is no automated comparison of the angular movements performed by patients on different days; (3) There is no visualization result and automated statistical computation to assist in the analysis and evaluation process of the patient's performance. Hence, a telehealth system using PoseNet-based system for in-home rehabilitation is proposed with the following advantages: (1) Using PoseNet with an ordinary webcam to detect the patients' angular movements incurs no additional expenditure on special-purpose equipment; (2) An automated comparison of the patients' angular movements on different days is built into the system; (3) A visualization result and statistical variables such as mean, standard deviation and the percentage deviation rate of the patients' angular movements are automatically calculated so that the doctors can analyze the patients' recovery status directly with more in-depth analysis.

\section{Proposed System}

In this section, the design and implementation of the proposed telehealth service using a PoseNet-based system for in-home rehabilitation is explained. The system architecture is presented, followed by the explanation on the computation of the body keypoints. Finally, the statistical computation for the patient's performance on different days is presented.

\subsection{System Architecture}

Figure 1 shows the system architecture of the proposed telehealth system. The system consists of three different modules namely, the account management module, the virtual appointment module and the prescription module. These modules are used by the patients, doctors and system administrators. 


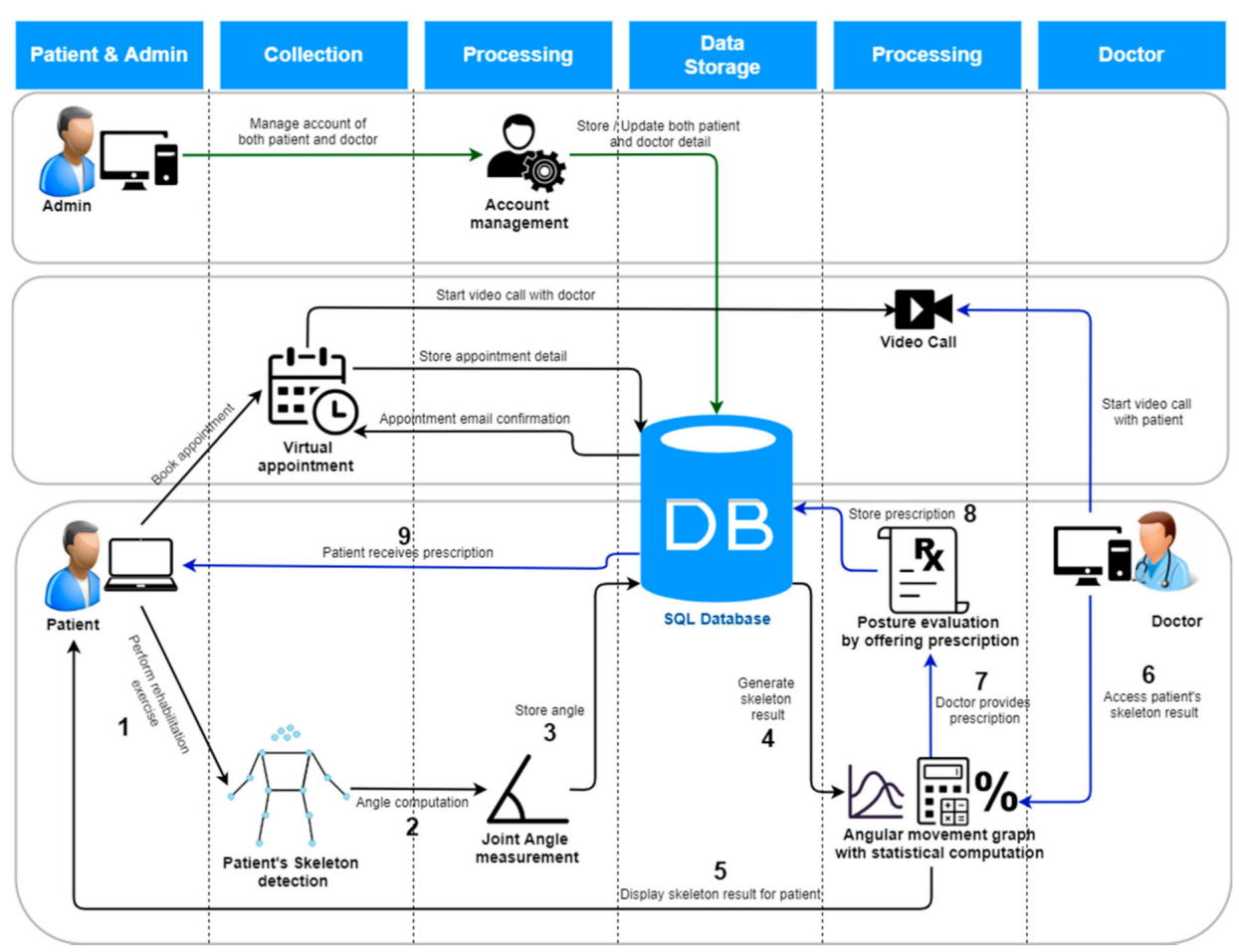

Figure 1. Proposed telehealth system architecture.

In the proposed system, the admins are responsible for maintaining the account of both patients and doctors. The patient can make virtual appointments with the doctor or perform the rehabilitation exercises, as shown in the bottom part of Figure 1. A patient can first schedule a virtual appointment with their doctor to discuss their physical condition. They will receive an appointment email notification when the doctor confirms the appointment schedule. During the scheduled appointment date and time, the patient will conduct a video call with the doctor. After discussing with the doctor, they can start the rehabilitation exercises (step 1). The proposed system will then calculate the patient's angular movements (step 2) and store them into the database (step 3). After completing the rehabilitation exercises, a skeleton result (angular movement graph) will be generated, enabling the patient (steps 4 and 5) to examine their angular movements through the graph. The doctors can then access the patient's data (step 6) to evaluate and compare the skeleton results on different selected days (step 7) to provide some advice or prescriptions to the patient (step 8). Finally, the patient can check the prescriptions and make adjustments to their subsequent exercises (step 9).

The skeleton detection method used to deploy the proposed system is the PoseNet. It is a machine learning model that can be applied for real-time human pose estimation [27,28]. By running PoseNet with Tensorflow.js library, anyone with camera-equipped devices such as laptops, PCs and smartphones can experience this technology directly from a web browser. The patient simply needs to stand in front of the device camera. The system will then track all the skeleton points of the patient. The PoseNet can detect a single pose or multiple poses from the images or videos within the browser. For our proposed system, only single-pose detection is implemented.

On accessing our proposed system, the patient firstly needs to log in to the web-based telehealth system, as shown in Figure 2a. The patient then needs to select one of their preferred doctors (Figure $2 b$ ) before performing rehabilitation exercises. Subsequently, the patient can click the start button in Figure $2 \mathrm{c}$ to perform the rehabilitation exercises. The system will start the 5-s preparation countdown timer before starting the video capture for the rehabilitation exercise. After completing the rehabilitation exercises, the skeleton result is instantly generated for patient and doctor. 


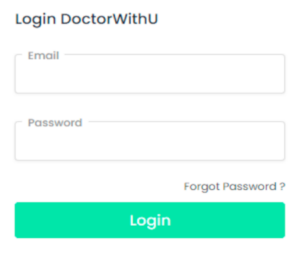

(a) Patient login

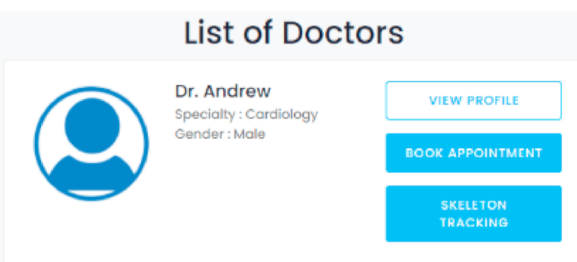

(b) List of doctors

Figure 2. Sample patient interface of the proposed telehealth system.

Figure 3 shows the skeleton detection processes during the rehabilitation exercise. First, the patient needs to connect his/her laptop to the internet connection to gain access to the web-based system. When the patient starts the rehabilitation session, their live video is recorded through the webcam and passed as an input to the PoseNet model. When the PoseNet model detects a human pose in the video frame, it extracts the keypoints of the patient by using the $2 \mathrm{D}$ coordinate $(\mathrm{x}, \mathrm{y})$ to indicate each detected body joint from every video frame. The extracted keypoints are used to calculate the distances and angles of both elbows and knees. When the predefined duration ends, the system will stop the recording and store all four angles from both elbows and knees as an array structure into the database. The patient and doctor can directly access the skeleton results. Based on the same set of actions performed by the patient during the few selected days, the doctor can examine the skeleton result by comparing and evaluating the deviation rate of the angular movements to determine the patient's recovery rate. Based on this, the doctor can offer advice or write a prescription for the patient.

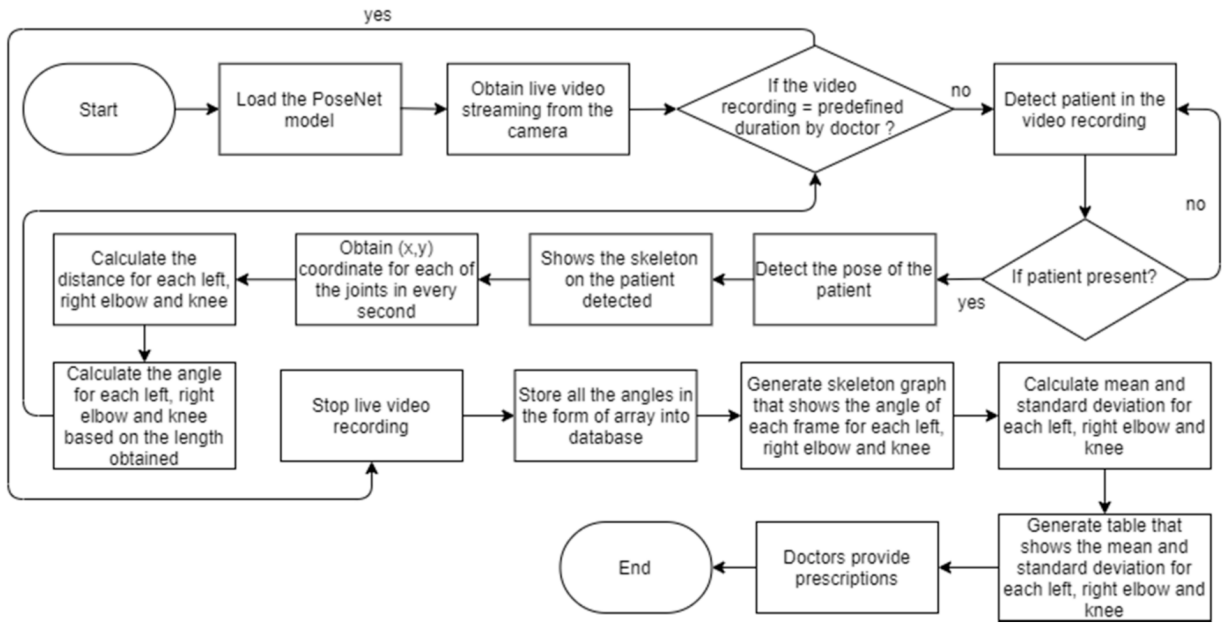

Figure 3. A detailed flowchart of the processes in the rehabilitation exercise.

In order for the doctor to obtain the patient's skeleton result, they first need log into the web-based telehealth system from the login page shown in Figure 4a. The system will then direct the doctor to the dashboard shown in Figure 4b. By selecting the All patients option at the My Patients (Skeleton) sidebar of Figure 4c, the doctor can view a pre-recorded 
skeleton list for all the patients. The doctor can then select one of the patient records by clicking the View patient's skeleton result/add patient's prescription orange button shown in Figure 4c. The skeleton result for that patient is generated instantly. From here, the doctor can compare the current and previous skeleton result by selecting two dates, as illustrated in Figure 4d. The doctor can then examine the patient's angular movements through the graph with the help of the built-in statistical computation shown in Figure 4f. After that, the doctor can advise or write a prescription for both elbow and knee movements of the patient. The advice and prescription from the doctor are directed to the specific patient in the format as illustrated in Figure 4e for any adjustment they should apply in their rehabilitation exercises.
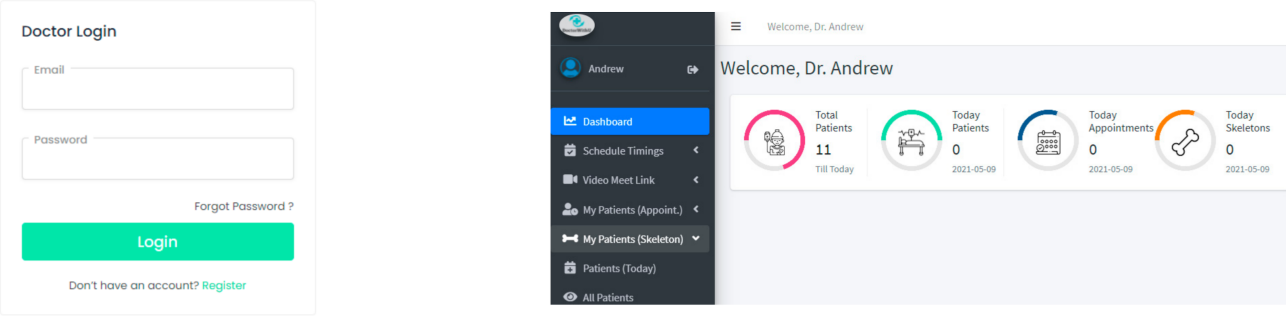

(a) Doctor login

(b) Dashboard
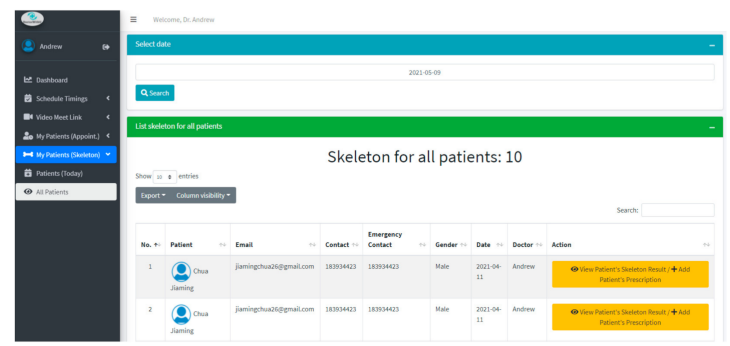

(c) Skeleton list for all patients
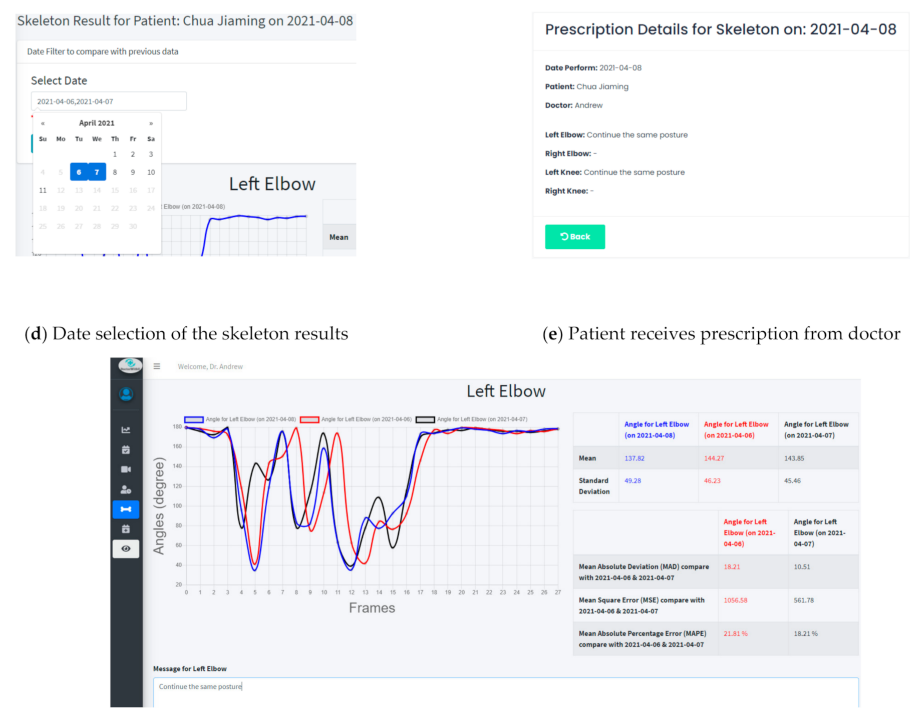

(f) Patient's skeleton result performed on three different days

Figure 4. Sample doctor interface of the proposed telehealth system.

Next, the administrators manage the accounts of patients and doctors, moderate the patients' appointment requests and verify the identities of all patients and doctors. After $\log$ into the web-based telehealth system (Figure 5a), the system will then direct the administrators to the dashboard shown in Figure 5b. By selecting Doctors and Patients 
option at the sidebar shown in Figure 5b, the administrators can view a list of doctors and patients. To manage the accounts for doctor or patient, the administrators can perform CRUD (create, read, update, delete) operations as illustrated in Figure $5 c$, d respectively. Besides, the administrators can also manage the patients' virtual appointment by selecting the Patient Appointment option at the sidebar shown in Figure $5 \mathrm{~b}$ to get a list of all patients' appointment shown in Figure 5e. To delete patient's appointment, the administrator can click the Cancel Appointment yellow button shown in Figure 5e.

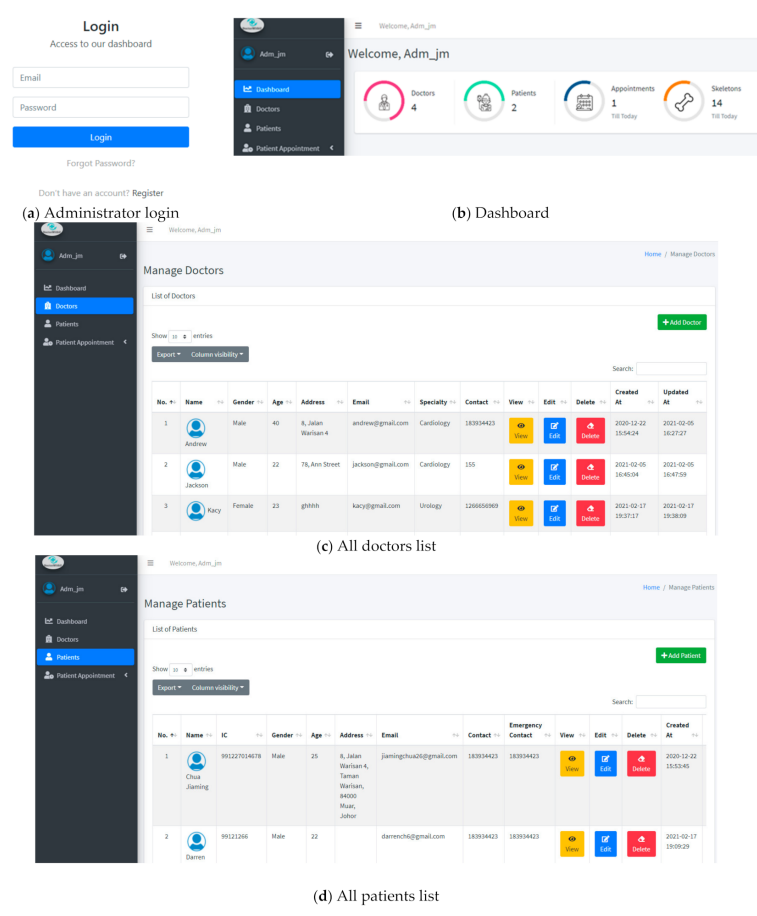

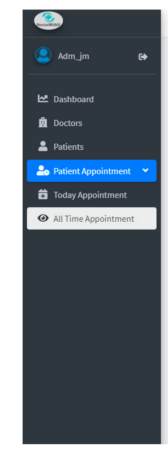

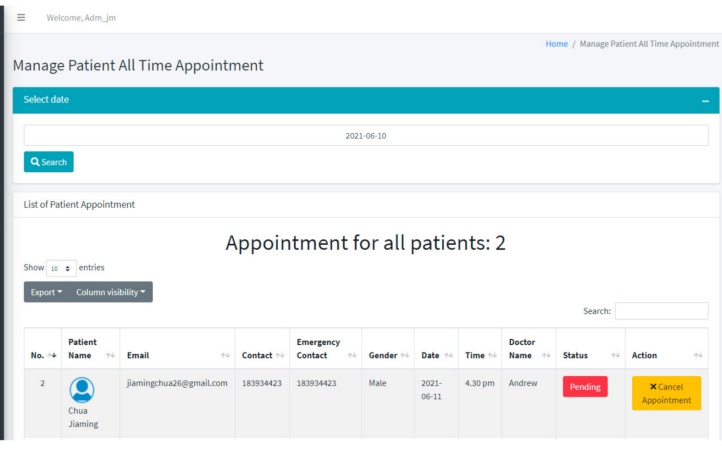

(e) All appointment patients list

Figure 5. Sample administrator interface of the proposed telehealth system.

\subsection{Body Keypoints Computation}

PoseNet can detect a total of 17 keypoints from different parts of the human body $[27,28]$. Each key point is represented by its $2 \mathrm{D}$ coordinate $(\mathrm{x}, \mathrm{y})$, as shown in Figure 6. During a rehabilitation session, the skeleton detection with 17 keypoints is displayed on the body. In the proposed system, only 12 keypoints are used to detect shoulders, elbows, wrists, hips, knees and ankles of the patient.

Figure 7 shows the angles for four body parts, namely the left hand, right hand, left leg and right leg, respectively. Each body part comprises three different keypoints, namely Point A (shoulders), Point B (elbows) and Point C (wrists) for both hands and Point A (hips), Point B (knees) and Point C (ankles) for both legs. Hence, both hands and legs comprise 12 keypoints in total. Each set of three keypoints in the four body parts are allocated with 2D coordinates, Point A (x1, y1), Point B (x2, y2) and Point C (x3, y3), respectively, forming the triangle shown in Figure 8. Next, the corresponding distances for Point A to Point B, Point B to Point $C$ and Point A to Point $C$ of the 12 keypoints are calculated for each video frame by using the Euclidean distance. 


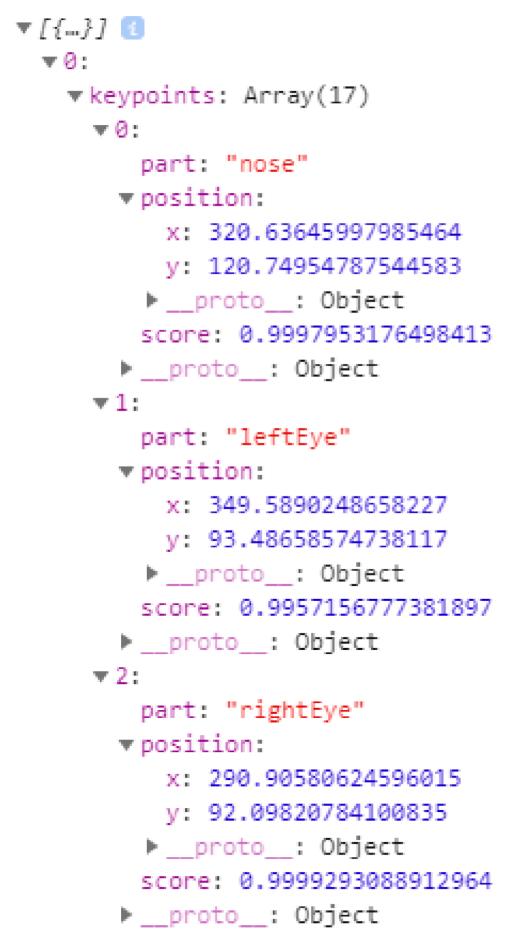

Figure 6. PoseNet keypoints in the form of $2 \mathrm{D}$ coordinate $(x, y)$.

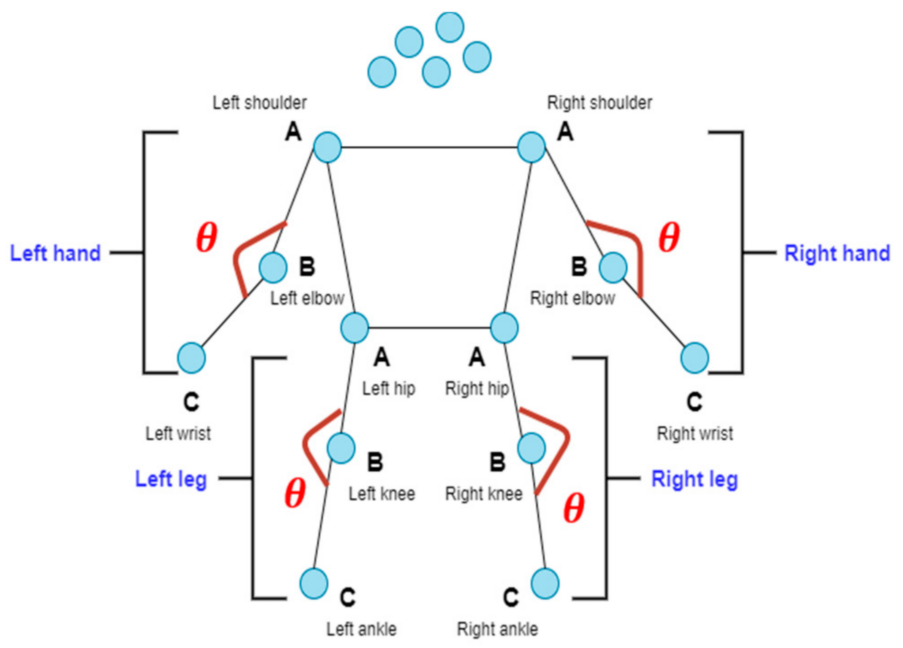

Figure 7. Angles on both elbows and knees.

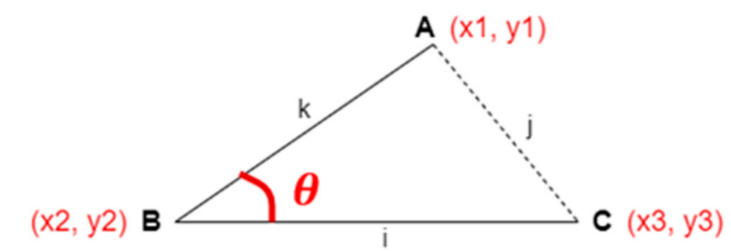

Figure 8. Angle in triangle representation.

Since each hand and leg is composed of three different keypoints, the angles of the keypoints are computed at Point B, which corresponds to both the elbow and knee positions of the patient. Since the flexion and extension exercises are usually performed on the elbows and knees, the computed angles will reflect the movement correctness to determine the performance of the flexion and extension exercises. After obtaining the distances from both hands and legs, the angles $\theta$ of both elbows and knees are calculated by substituting the value of $i, j$ and $k$ into the $\arccos ()$ function. 
Finally, the skeleton result is generated for both patient and doctor to examine the angular movements. Meanwhile, the Mean, $\mu$ and Standard Deviation, $\sigma$ are computed to display the average of the angular movements and to show how much the angles in a distribution spread out from one another. A low value of $\sigma$ indicates that the angular movements from each frame are clustered closely around the $\mu$, whereas a high value of $\sigma$ indicates the angular movements are spreading to a wider range.

\subsection{Statistical Computation}

After the patient has performed the same rehabilitation exercises on the selected days, the doctor can compare the patient's angular movements between the different days by referring to the Mean Absolute Deviation (MAD), Mean Squared Error (MSE) and Mean Absolute Percentage Error (MAPE), as defined in Equations (1)-(3). These statistical computations are very useful to assist in the evaluation process because they offer a more-in-depth analysis metrics for the doctor to write their prescription.

$M A D$ is used to calculate the average distance between the current and previous skeleton results for each angle point of each frame, whereas the MSE is used to calculate the average squared difference. Lastly, the MAPE displays the percentage deviation rate of the current skeleton result as compared to the previous skeleton result. These three formulae are commonly used in measuring the forecasting performance [29-31]. MSE is more sensitive to errors than $M A D$ because all forecast errors are squared, which causes the value of $M S E$ to always be greater than $M A D$. Therefore, the forecast error in $M S E$ becomes more pronounced. However, the major shortcoming of MAD and MSE is that they do not consider the magnitude of the actual value [29]. As a result, the forecast error does not reflect the accuracy of the angular movements. Hence, MAPE is applied to address this issue as it takes into account the magnitude of the actual values. It sums up all the absolute errors for each period with respect to actual values and then expresses the average error in term of generic percentage. This is useful for reporting purposes in our proposed system as it can be used to determine a patient's recovery rate. This is because the average accumulated error rate in $M A P E$ corresponds to the patient's angular data in each frame as compared to the actual values. The lower values of $M A D, M S E$ and MAPE signify a more accurate model, which indicates that the postural movements performed by the patient are relatively better.

The low values of $M A D$ and MSE show that the average and squared error of the patient's angular movements in each frame is small as compared to the actual data from the physician's movements. Similarly, a low value of MAPE indicates that the average accumulation error percentage of the patient's angular movements in each frame has a small deviation rate. Most importantly, the MAPE plays a crucial role because the doctor can further analyze the patient's recovery status through the percentage deviation rate to determine whether the patient's condition has improved.

$$
\begin{gathered}
M A D=\frac{\sum_{t=1}^{N}\left|A_{t}-F_{t}\right|}{N} \\
M S E=\frac{\sum_{t=1}^{N}\left(A_{t}-F_{t}\right)^{2}}{N} \\
M A P E=\frac{\sum_{t=1}^{N}\left|\frac{A_{t}-F_{t}}{A_{t}}\right|}{N} \times 100 \%
\end{gathered}
$$

where $A_{t}$ is the actual value from the patient's movement, $F_{t}$ is the value from the benchmark movement, and $N$ is the total number of frames.

\section{Methodology}

In order to validate the functionalities of the automated statistical computation to assist in the analysis and evaluation process of the patient's performance in the proposed system, several functional tests were executed. One subject of 22 years old male and one subject of 
20 years old female were invited to participate in the functional tests. Both subjects were required to stand in front of the laptop webcam to perform the rehabilitation exercises, as shown Figure 9. A series of benchmark poses were selected from the well-known KIT dataset [32] to represent the ground-truth skeleton-angular movements to be compared to those movements performed by both subjects. The KIT Whole-Body Human Motion Database is a large-scale dataset that offers a data corpus of 2615 different movements, focusing not only on mere whole-body motions but also on whole-body actions (this study makes use only of the motions).

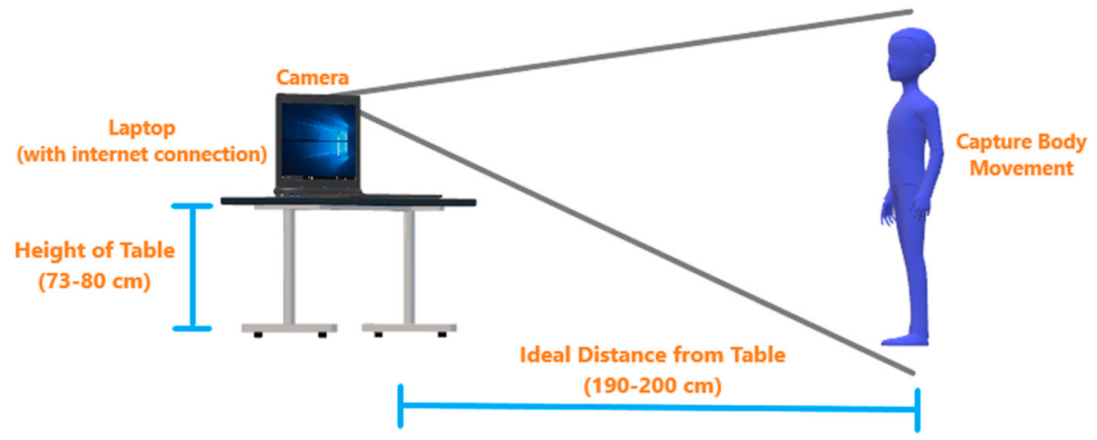

Figure 9. Functional test setup.

Since the invited subjects did not have any previous experience in rehabilitation exercises, two functional tests were conducted. The purpose of conducting these two tests was to compare the different postures of hand and leg movements performed by both subjects to those from the KIT dataset. Both subjects were required to follow the selected movements from the KIT dataset for two selected days. The first video clip guides the subjects to demonstrate both arms lifted at 90 degrees and to bend to the front for a duration of $30 \mathrm{~s}$, as illustrated in Figure 10. This set of movements helps to enhance the body posture and strengthen the muscles of the shoulders and arms. The second video clip guides the subjects to perform the half squatting action for $20 \mathrm{~s}$, as shown in Figure 11. This set of movements increases the stability of the hip abductor, thus stabilizing the pelvis and offering good balancing training. In order to gain a fair comparison between the benchmark poses and subjects' poses, both subjects performed the set of movements in the first and second tests for 30 and 20 s respectively, following the durations of the original benchmark movements.

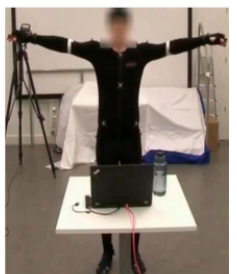

(a) Frame 1

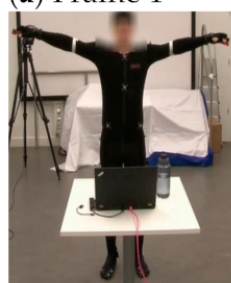

(g) Frame 116

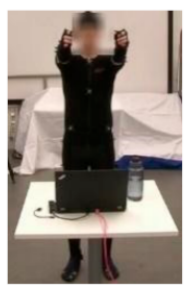

(b) Frame 16

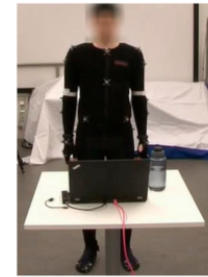

(h) Frame 130

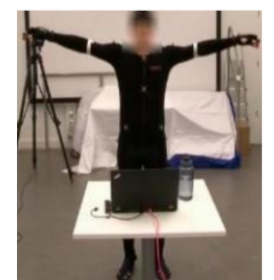

(c) Frame 49

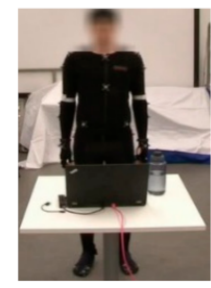

(d) Frame 54

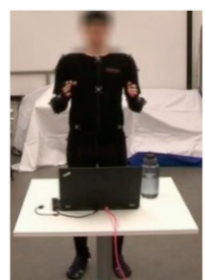

(e) Frame 76

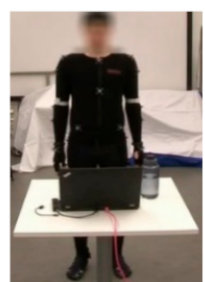

(f) Frame 86

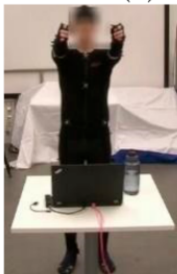

(j) Frame 160

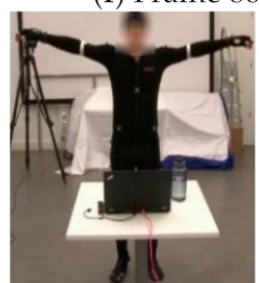

(k) Frame 173

Figure 10. Video clip 1: both arms lifted in 90 degrees and bend to the front. 


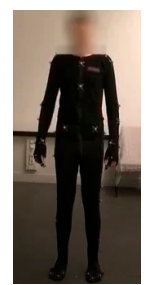

(a) Frame 1

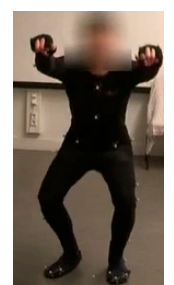

(b) Frame 5

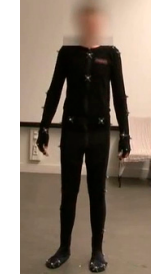

(c) Frame 13

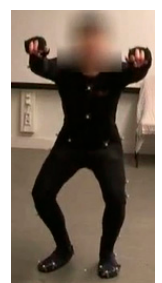

(d) Frame 32

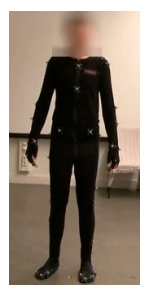

(e) Frame 43

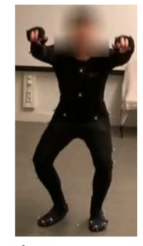

(f) Frame 62

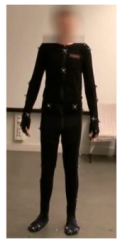

(g) Frame 73

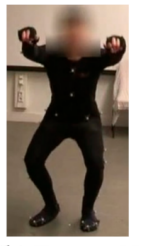

(h) Frame 92

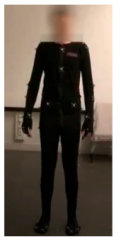

(i) Frame 108

Figure 11. Video clip 2: half squatting.

\section{Results and Discussion}

Figures 12 and 13 show the skeleton detection in both of the video clips of the benchmark poses. The benchmark angular movements from the KIT dataset were calculated.
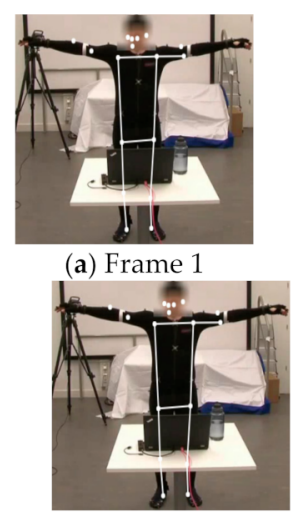

(g) Frame 116

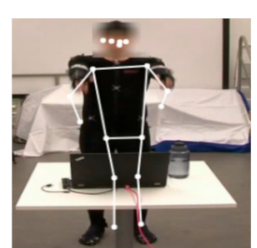

(b) Frame 16

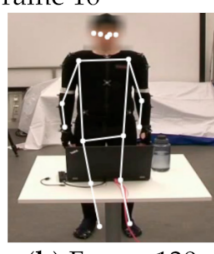

(h) Frame 130

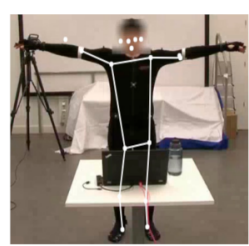

(c) Frame 49

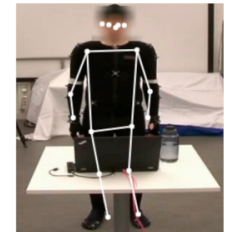

(d) Frame 54

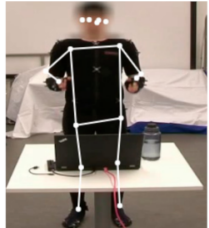

(e) Frame 76

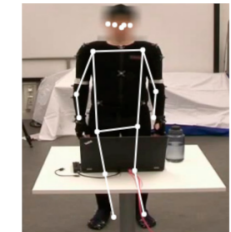

(f) Frame 86

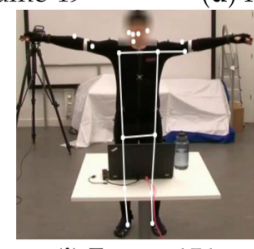

(i) Frame 151

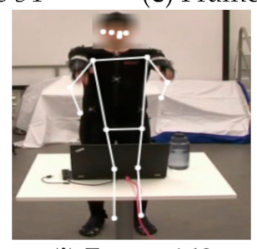

(j) Frame 160

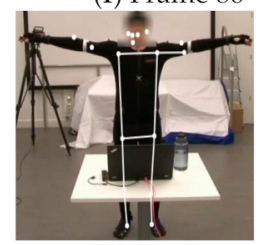

(k) Frame 173

Figure 12. Skeleton detection for the benchmark poses in video clip 1.

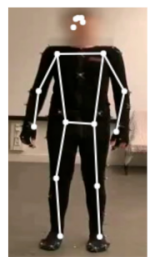

(a) Frame 1

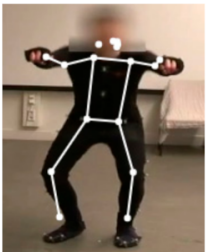

(f) Frame 62

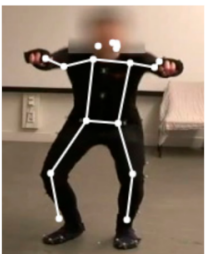

(b) Frame 5

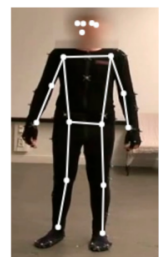

(c) Frame 13

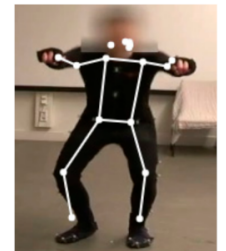

(d) Frame 32

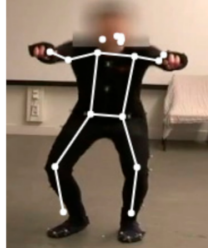

(h) Frame 92

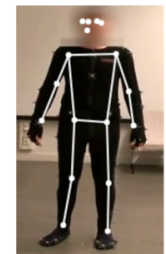

(e) Frame 43

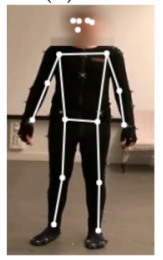

(i) Frame 108

Figure 13. Skeleton detection for the benchmark poses in video clip 2.

Figure 14a,e show the posture of the left elbow as performed by both subjects in the first functional test. The dotted region of the angular movements graph shows the obvious angular differences between Day 1 (red line) and Day 2 (black line) as compared to the 
benchmark angular movements from the KIT dataset (blue line). Subsequently, the MAPE values of the left elbow movements as performed by subject 1 are shown in Table 1 with $51.71 \%$ (Day 1) and 34.99\% (Day 2), respectively. On the contrary, subject 2 performed better in terms of the posture of the left elbow as compared to subject 1 on Day 1 and Day 2 . Hence, the MAPE values of the left elbow performed by subject 2, shown in Table 1 with $19.68 \%$ (Day 1) and $11.01 \%$ (Day 2), are significantly lower than those of subject 1 . In spite of this, there is slight improvement in the posture of the left elbow performed by both subjects on Day 2 as compared to Day 1, which is reflected in the decreased MAPE values.

Angular movement (KIT dataset)

$\square$ Day 1 (Subject's angular movement)

$\square$ Day 2 (Subject's angular movement)

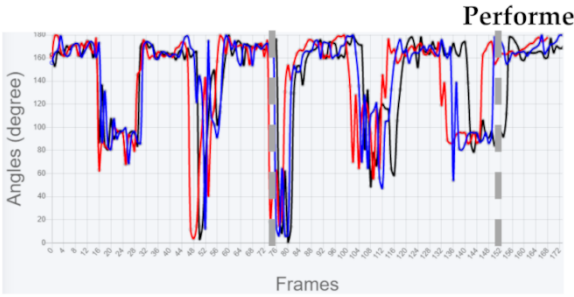

(a) Left elbow

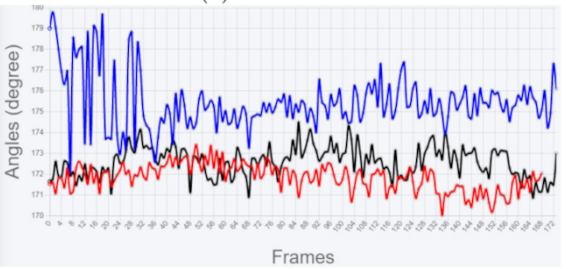

(c) Left knee Perfomed by Subject 2 (Female)

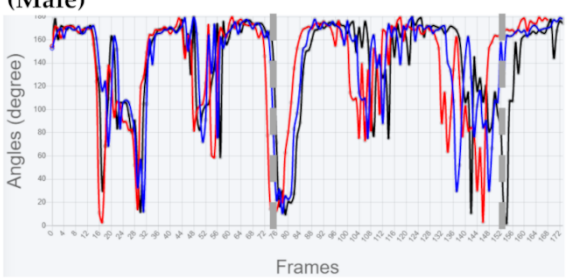

(b) Right elbow

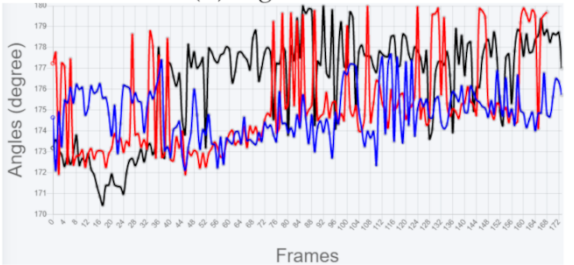

(d) Right knee

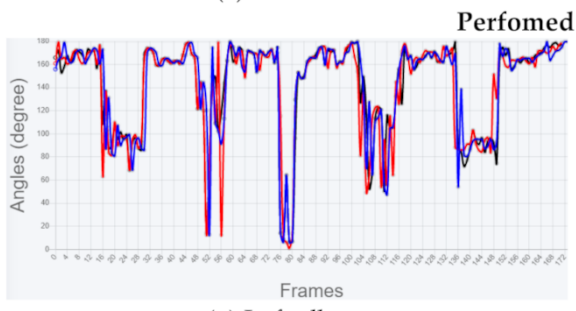

(e) Left elbow

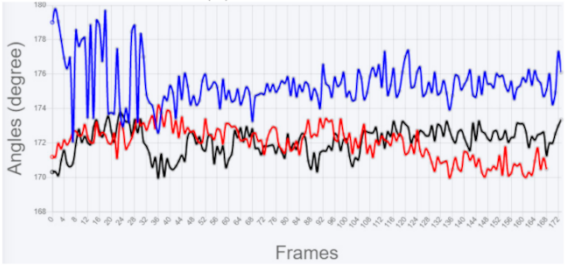

(g) Left Knee

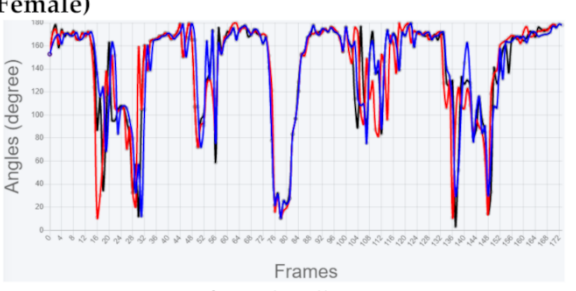

(f) Right elbow

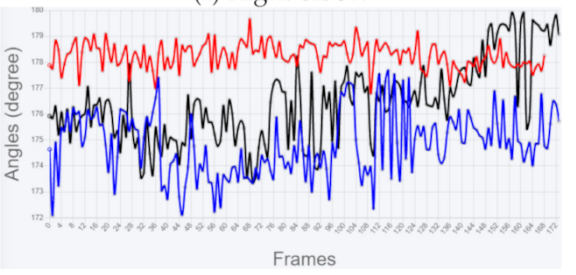

(h) Right Knee

Figure 14. Angular movements graph for functionality test 1 .

For the right elbow shown in Figure $14 \mathrm{~b}, \mathrm{f}$, subject 1 shows obvious angle differences in the dotted region of the angular movements graph for both Day 1 and Day 2 due to the incorrect performance of the right-arm-lifting posture. These differences are reflected in the MAPE values of Table 1 for the right elbow in Day 1 and Day 2 of $35.75 \%$ and 28.55\%, respectively. Subject 2 still performed relatively well for the posture of the right elbow, similar to that of the left elbow. Hence, the MAPE values of the right elbow movement of subject 2 are comparatively lower as shown in Table 1 with $19.76 \%$ (Day 1) and 13.58\% (Day 2), respectively. Meanwhile, both subjects' angular movements are consistent for both knees, as shown in Figure 14c,d,g,h, hovering between 170 to 180 degrees. Since both legs simply stand still in this sequence of poses, the angular movements of left and right knees are expected to maintain consistently low MAPE values. 
Table 1. Mean, Standard Deviation, MAD, MSE and MAPE for functionality test 1.

\begin{tabular}{|c|c|c|c|c|c|c|c|c|}
\hline & & & \multirow{2}{*}{$\begin{array}{l}\text { Angular Movement } \\
\text { (KIT Dataset) }\end{array}$} & \multicolumn{2}{|c|}{$\begin{array}{l}\text { Subject's Angular } \\
\text { Movement }\end{array}$} & & \multicolumn{2}{|c|}{$\begin{array}{c}A_{t}=\text { KIT Angular Movement, } \\
F_{t}=\text { Subject's Angular Movement }\end{array}$} \\
\hline & & & & Day 1 & Day 2 & & $\begin{array}{l}\text { Deviation Rate } \\
\text { (Day 1) }\end{array}$ & $\begin{array}{l}\text { Deviation Rate } \\
\text { (Day 2) }\end{array}$ \\
\hline \multirow{12}{*}{ Subject 1 (Male) } & \multirow{3}{*}{ Left Elbow } & Mean, $\mu$ & 143.04 & 140.83 & 142.83 & MAD & 24.27 & 21.81 \\
\hline & & \multirow{2}{*}{$\begin{array}{c}\text { Standard } \\
\text { Deviation, } \sigma\end{array}$} & \multirow{2}{*}{41.09} & \multirow{2}{*}{43.06} & \multirow{2}{*}{41.49} & MSE & 1834.36 & 1403.42 \\
\hline & & & & & & MAPE & $51.71 \%$ & $34.99 \%$ \\
\hline & \multirow{3}{*}{ Right Elbow } & Mean, $\mu$ & 142.25 & 138.13 & 141.1 & MAD & 26.78 & 22.7 \\
\hline & & Standard & \multirow{2}{*}{42.59} & \multirow{2}{*}{46.37} & \multirow{2}{*}{44.34} & MSE & 1715.62 & 1399.89 \\
\hline & & Deviation, $\sigma$ & & & & MAPE & $35.75 \%$ & $28.55 \%$ \\
\hline & \multirow{3}{*}{ Left Knee } & Mean, $\mu$ & 175.46 & 171.83 & 172.58 & MAD & 3.62 & 2.88 \\
\hline & & Standard & \multirow{2}{*}{1.35} & \multirow{2}{*}{0.64} & \multirow{2}{*}{1.05} & MSE & 15.63 & 10.86 \\
\hline & & Deviation, $\sigma$ & & & & MAPE & $2.06 \%$ & $1.64 \%$ \\
\hline & \multirow{3}{*}{ Right Knee } & Mean, $\mu$ & 174.83 & 175.14 & 176.52 & MAD & 1.91 & 2.87 \\
\hline & & Standard & \multirow{2}{*}{1.23} & \multirow{2}{*}{2.25} & \multirow{2}{*}{2.72} & MSE & 6.3 & 10.25 \\
\hline & & Deviation, $\sigma$ & & & & MAPE & $1.09 \%$ & $1.64 \%$ \\
\hline \multirow{12}{*}{ Subject 2 (Female) } & \multirow{3}{*}{ Left Elbow } & Mean, $\mu$ & 143.04 & 141.08 & 142.52 & MAD & 13.95 & 9.2 \\
\hline & & Standard & \multirow{2}{*}{41.09} & \multirow{2}{*}{42.41} & \multirow{2}{*}{40.74} & MSE & 673.99 & 375.88 \\
\hline & & Deviation, $\sigma$ & & & & MAPE & $19.68 \%$ & $11.01 \%$ \\
\hline & \multirow{3}{*}{ Right Elbow } & Mean, $\mu$ & 142.25 & 139.98 & 140.55 & MAD & 16.02 & 10.22 \\
\hline & & Standard & \multirow{2}{*}{42.59} & \multirow{2}{*}{44.74} & \multirow{2}{*}{44.48} & MSE & 815.41 & 384.23 \\
\hline & & Deviation, $\sigma$ & & & & MAPE & $19.76 \%$ & $13.58 \%$ \\
\hline & \multirow{3}{*}{ Left Knee } & Mean, $\mu$ & 175.46 & 171.99 & 172.08 & MAD & 3.49 & 3.39 \\
\hline & & Standard & \multirow{2}{*}{1.35} & 095 & ( 82 & MSE & 15.03 & 13.79 \\
\hline & & Deviation, $\sigma$ & & 0.90 & 0.02 & MAPE & $1.98 \%$ & $1.92 \%$ \\
\hline & & Mean, $\mu$ & 174.83 & 178.26 & 176.52 & MAD & 3.46 & 1.89 \\
\hline & Right Knee & Standard & $12 ?$ & (1) & 200 & MSE & 13.79 & 5.42 \\
\hline & & Deviation, $\sigma$ & 1.23 & 0.48 & 2.38 & MAPE & $1.98 \%$ & $1.08 \%$ \\
\hline
\end{tabular}

On the contrary, the angular movements graph in the second functional test performed by both subjects show significant angle differences, which are highlighted by the dotted line portion in Figure 15a,e. These are also reflected in the left elbow of Table 2 with MAPE values of $12.61 \%$ (day 1 ) and $12.31 \%$ (day 2 ) for subject 1 and $13.53 \%$ (day 1 ) and $10.73 \%$ (day 2) for subject 2. Subsequently, subject 2 shows significant improvement for the posture of right elbow from Day 1 to Day 2 with decreased MAPE values (19.41\% to $14.49 \%$ ) as compared to subject 1 . The angular movements graph for both knees performed by both subjects shows minor variations for both days, as illustrated in Figure 15c,d,g,h with MAPE values ranging from $4 \%$ to $7 \%$. Moreover, the movements for both knees performed by both subjects on Day 2 are improving based on the decreasing MAPE values. The low MAPE values of the left and right knees also indicate that both subjects had followed the half squatting pose closely compared to the benchmark pose from the KIT dataset. 


\section{Performed by Subject 1 (Male)}

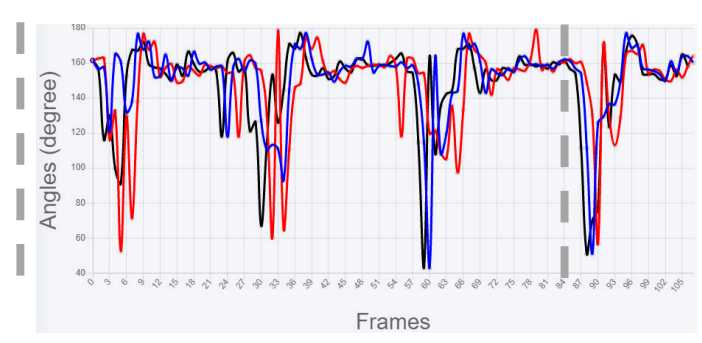

(a) Left elbow

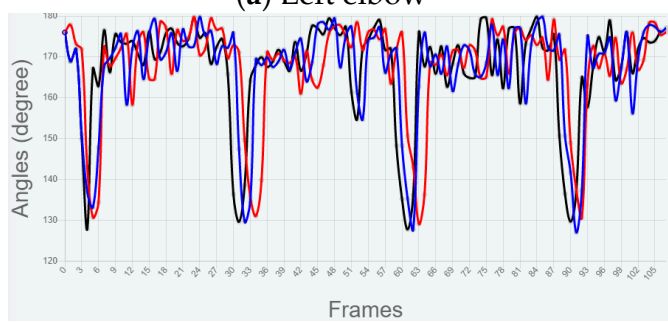

(c) Left knee

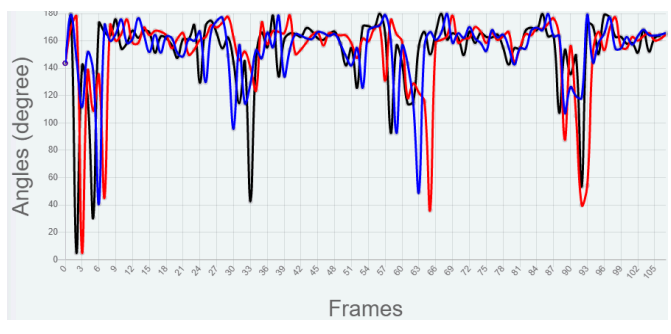

(b) Right elbow

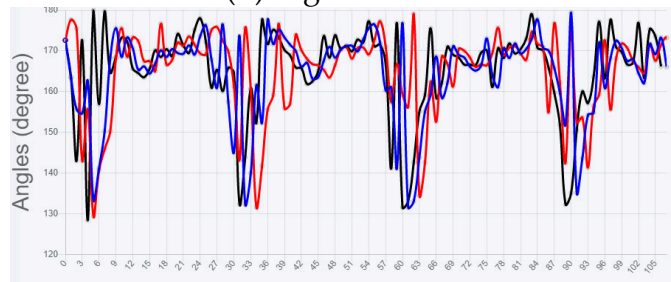

Frames

(d) Right knee

Performed by subject 2 (Female)

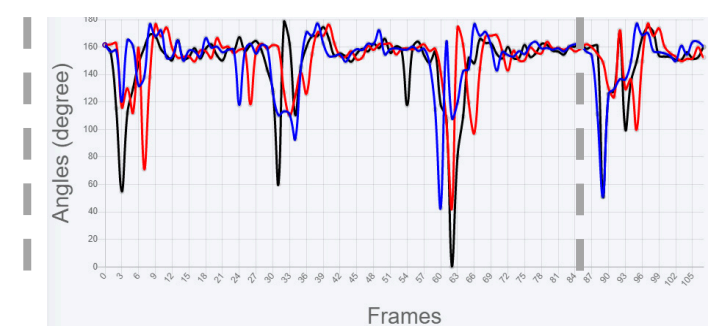

(e) Left elbow

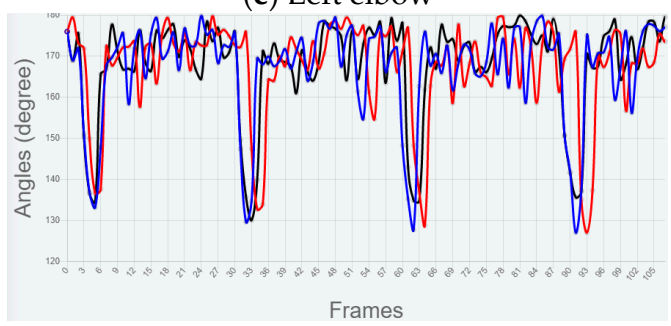

(g) Left knee

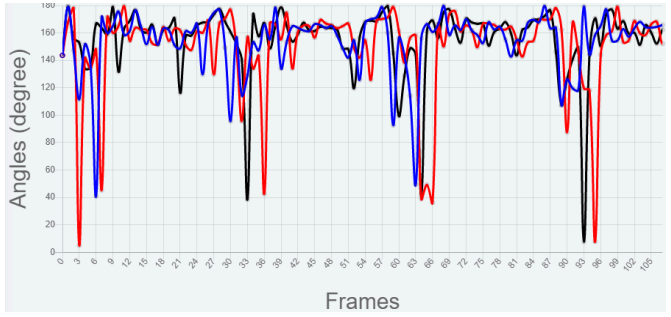

(f) Right elbow

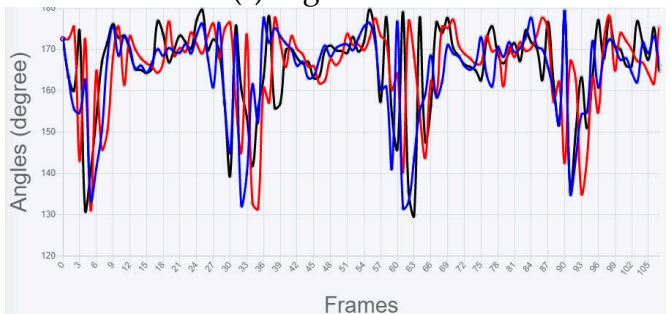

(h) Right knee

Figure 15. Angular movements graph for functionality test 2.

In summary, both functional tests have demonstrated the PoseNet-based system for in-home rehabilitation with the following validations: (1) using PoseNet with an ordinary webcam to detect the patients' angular movements; (2) an automated comparison of the patients' angular movements on different days is instantly available for patients and doctors after the rehabilitation exercise; (3) visualization graph and statistical computation is automatically generated for doctors to examine the patients' recovery rate with a more in-depth analysis. Although the functionality of the skeleton detection is validated to offer the angular movement graph and statistical computation for the doctor's evaluation, there are two limitations that need to be considered in the proposed system. First, the patient needs to ensure that no other human is visible within the webcam view during the rehabilitation session as this will lead to inaccurate skeleton detection. Second, the patient needs to ensure that his/her laptop is placed following the recommended standing distance as specified in the system setup (Figure 9). This will ensure that the webcam can capture the patient's entire body in the camera view for accurate analysis. 
Table 2. Mean, Standard Deviation, MAD, MSE and MAPE for functionality test 2.

\begin{tabular}{|c|c|c|c|c|c|c|c|c|}
\hline & & & \multirow{2}{*}{$\begin{array}{l}\text { Angular Movement } \\
\text { (KIT Dataset) }\end{array}$} & \multicolumn{2}{|c|}{$\begin{array}{l}\text { Subject's Angular } \\
\text { Movement }\end{array}$} & & \multicolumn{2}{|c|}{$\begin{array}{c}A_{t}=\text { KIT Angular Movement } \\
F_{t}=\text { Subject's Angular Movement }\end{array}$} \\
\hline & & & & Day 1 & Day 2 & & $\begin{array}{c}\text { Deviation Rate } \\
\text { (Day 1) }\end{array}$ & $\begin{array}{l}\text { Deviation Rate } \\
\text { (Day 2) }\end{array}$ \\
\hline \multirow{12}{*}{ Subject 1 (Male) } & \multirow{3}{*}{ Left Elbow } & Mean, $\mu$ & 150.96 & 148.17 & 148.37 & MAD & 14.94 & 14.25 \\
\hline & & \multirow{2}{*}{$\begin{array}{c}\text { Standard } \\
\text { Deviation, } \sigma\end{array}$} & \multirow{2}{*}{21.68} & \multirow{2}{*}{25.07} & \multirow{2}{*}{25.09} & MSE & 630.94 & 616.6 \\
\hline & & & & & & MAPE & $12.61 \%$ & $12.31 \%$ \\
\hline & \multirow{3}{*}{ Right Elbow } & Mean, $\mu$ & 154.04 & 152.75 & 153.06 & MAD & 18.21 & 18.72 \\
\hline & & Standard & \multirow{2}{*}{23.04} & \multirow{2}{*}{30.19} & \multirow{2}{*}{28.63} & MSE & 1041.18 & 1045.65 \\
\hline & & Deviation, $\sigma$ & & & & MAPE & $15.23 \%$ & $16.75 \%$ \\
\hline & \multirow{3}{*}{ Left Knee } & Mean, $\mu$ & 167.14 & 167.64 & 167.35 & MAD & 7.85 & 7.15 \\
\hline & & Standard & \multirow{2}{*}{12.35} & \multirow{2}{*}{12.31} & \multirow{2}{*}{12.56} & MSE & 125.68 & 106.07 \\
\hline & & Deviation, $\sigma$ & & & & MAPE & $4.85 \%$ & $4.46 \%$ \\
\hline & \multirow{3}{*}{ Right Knee } & Mean, $\mu$ & 164.57 & 164.84 & 165.3 & MAD & 7.78 & 7.78 \\
\hline & & Standard & \multirow{2}{*}{10.79} & \multirow{2}{*}{10.59} & \multirow{2}{*}{11.16} & MSE & 132.22 & 161.8 \\
\hline & & Deviation, $\sigma$ & & & & MAPE & $4.94 \%$ & $4.92 \%$ \\
\hline \multirow{12}{*}{ Subject 2 (Female) } & \multirow{3}{*}{ Left Elbow } & Mean, $\mu$ & 150.96 & 151.02 & 148.51 & MAD & 15.6 & 13 \\
\hline & & Standard & \multirow{2}{*}{21.68} & \multirow{2}{*}{20.65} & \multirow{2}{*}{26.84} & MSE & 689.02 & 543.08 \\
\hline & & Deviation, $\sigma$ & & & & MAPE & $13.53 \%$ & $10.73 \%$ \\
\hline & \multirow{3}{*}{ Right Elbow } & Mean, $\mu$ & 154.04 & 149.75 & 155.36 & MAD & 23.57 & 16.11 \\
\hline & & Standard & \multirow{2}{*}{23.04} & \multirow{2}{*}{34.41} & \multirow{2}{*}{26.53} & MSE & 1649.98 & 988.74 \\
\hline & & Deviation, $\sigma$ & & & & MAPE & $19.41 \%$ & $14.49 \%$ \\
\hline & \multirow{3}{*}{ Left Knee } & Mean, $\mu$ & 167.14 & 167.63 & 168 & MAD & 10.27 & 5.34 \\
\hline & & Standard & \multirow{2}{*}{12.35} & 1205 & 1205 & MSE & 231.15 & 66.6 \\
\hline & & Deviation, $\sigma$ & & 12.05 & 12.05 & MAPE & $6.45 \%$ & $3.27 \%$ \\
\hline & & Mean, $\mu$ & 164.57 & 165.82 & 166.01 & MAD & 8.58 & 6.32 \\
\hline & Right Knee & Standard & 1070 & 1047 & 1073 & MSE & 145.14 & 103.96 \\
\hline & & Deviation, $\sigma$ & 10.19 & 10.47 & $10 . / 3$ & MAPE & $5.44 \%$ & $4.01 \%$ \\
\hline
\end{tabular}

\section{Conclusions}

This paper presents a telehealth service using the PoseNet-based system for in-home rehabilitation using an ordinary webcam. The proposed system offers benefits for patients in performing daily rehabilitation through remote monitoring. This is especially useful for those elderly patients with limited mobility and chronic condition. The system is also useful to reduce the travelling challenges for those patients from rural areas who need to perform daily rehabilitation exercises. Using an ordinary webcam for rehabilitation is very promising because it enables the patients to adopt in-home rehabilitation without additional cost.

The proposed system is validated to operate well and is able to detect the variation of the subject's movements in both functional tests. Since PoseNet is completely free and can successfully capture all the skeleton points, PoseNet may be a more economical solution than the Microsoft Kinect for in-home rehabilitation system, helping to reduce the patient's financial burden. In addition, the built-in statistical computation plays a crucial role in the rehabilitation procedure to assist the doctors in performing deeper analysis in determining the patient's recovery rate. The functional tests also demonstrated the usefulness of the statistical results for the subjects to monitor their progress on different days.

Having completed functional tests, the system is ready for clinical trials that will be carried out in collaboration with the rehabilitation centers to evaluate the proposed telehealth system for actual in-home rehabilitation practices. The clinical trials will further investigate the effectiveness of the PoseNet-based in-home rehabilitation to meet the patients' needs while also maintaining patient safety, study integrity, and data quality. Subsequent future work includes introducing a 3D avatar that can demonstrate the rehabilitation exercises 
more vividly and provide guidance on how to perform them more accurately. On top of this, background music can be added to make the rehabilitation exercise more appealing and relaxing for patients.

Author Contributions: Methodology, J.C.; data curation, J.C.; writing-original draft preparation, J.C.; writing-review and editing, L.-Y.O.; visualization, J.C.; supervision, L.-Y.O.; project administration, M.-C.L.; funding acquisition, M.-C.L. All authors have read and agreed to the published version of the manuscript.

Funding: This research was funded by Telekom Malaysia Research \& Development, grant number RDTC/191001 (MMUE/190086) and Multimedia University.

Data Availability Statement: The data presented in this study are available on request from the corresponding author.

Conflicts of Interest: The authors declare no conflict of interest.

\section{References}

1. Catalyst, N.E.J.M. What Is Telehealth? NEJM Catalyst. Available online: https://catalyst.nejm.org/doi/full/10.1056/CAT.18.026 8/ (accessed on 12 June 2021).

2. Wosik, J.; Fudim, M.; Cameron, B.; Gellad, Z.F.; Cho, A.; Phinney, D.; Curtis, S.; Roman, M.; Poon, E.G.; Ferranti, J.; et al. Telehealth Transformation: COVID-19 and the Rise of Virtual Care. J. Am. Med Inform. Assoc. 2020, 27, 957-962. [CrossRef] [PubMed]

3. "Ageing and Health". World Health Organization. Available online: https://www.who.int/news-room/fact-sheets/detail/ ageing-and-health/ (accessed on 12 June 2021).

4. Bate, N.J.; Xu, S.C.; Pacilli, M.; Roberts, L.J.; Kimber, C.; Nataraja, R.M. Effect of the COVID-19 Induced Phase of Massive Telehealth Uptake on End-User Satisfaction. Intern. Med. J. 2021, 51, 206-214. [CrossRef] [PubMed]

5. Boissy, P.; Brière, S.; Corriveau, H.; Grant, A.; Lauria, M.; Michaud, F. Usability testing of a mobile robotic system for in-home telerehabilitation. In Proceedings of the 2011 Annual International Conference of the IEEE Engineering in Medicine and Biology Society, Boston, MA, USA, 30 August-3 September 2011. [CrossRef]

6. Rogante, M.; Grigioni, M.; Cordella, D.; Giacomozzi, C. Ten years of telerehabilitation: A literature overview of technologies and clinical applications. NeuroRehabilitation 2010, 27, 287-304. [CrossRef] [PubMed]

7. Tousignant, M.; Boissy, P.; Moffet, H.; Corriveau, H.; Cabana, F.; Marquis, F.; Simard, J. Patients' Satisfaction of Healthcare Services and Perception with In-Home Telerehabilitation and Physiotherapists' Satisfaction Toward Technology for Post-Knee Arthroplasty: An Embedded Study in a Randomized Trial. Telemed. e-Health 2011, 17, 376-382. [CrossRef] [PubMed]

8. Tousignant, M.; Moffet, H.; Boissy, P.; Corriveau, H.; Cabana, F.; Marquis, F. A randomized controlled trial of home telerehabilitation for post-knee arthroplasty. J. Telemed. Telecare 2011, 17, 195-198. [CrossRef] [PubMed]

9. Tay, E.L.; Wong, C.P. A Cross-Sectional Survey of Rehabilitation Service Provision for Children with Brain Injury in Selangor, Malaysia. Disabil. CBR Incl. Dev. 2018, 29, 45-58. [CrossRef]

10. Nitta, Y.; Murayama, Y. Privacy-Aware Remote Monitoring System by Skeleton Recognition. In Proceedings of the 52nd Hawaii International Conference on System Sciences, Maui, HI, USA, 8-11 January 2019. [CrossRef]

11. Robert-Lachaine, X.; Mecheri, H.; Muller, A.; Larue, C.; Plamondon, A. Validation of a Low-Cost Inertial Motion Capture System for Whole-Body Motion Analysis. J. Biomech. 2020, 99, 109520. [CrossRef] [PubMed]

12. Household Income \& Basic Amenities Survey Report 2019, Department of Statistics Malaysia Official Portal. Available online: https:/ / www.dosm.gov.my/v1/index.php?r=column/cthemeByCat\&cat=120\&bul_id=TU00TmRhQ1N5TUxHVWN0T2 VjbXJYZz09\&menu_id=amVoWU54UT10a21NWmdhMjFMMWcyZz09 (accessed on 18 March 2021).

13. Ong, A.; Harris, I.S.; Hamill, J. The Efficacy of a Video-Based Marker-Less Tracking System for Gait Analysis. Comput. Methods Biomech. Biomed. Eng. 2017, 20, 1089-1095. [CrossRef] [PubMed]

14. Samad, R.; Abu Bakar, M.Z.; Pebrianti, D.; Mustafa, M.; Abdullah, N.R.H. Elbow Flexion and Extension Rehabilitation Exercise System Using Marker-Less Kinect-Based Method. Int. J. Electr. Comput. Eng. (IJECE) 2017, 7, 1602-1610. [CrossRef]

15. Kendall, A.; Grimes, M.; Cipolla, R. Posenet: A Convolutional Network for Real-Time 6-dof Camera Relocalization. In Proceedings of the IEEE International Conference on Computer Vision, Santiago, Chile, 7-13 December 2015; pp. 2938-2946. [CrossRef]

16. Anton, D.; Goni, A.; Illarramendi, A.; Torres-Unda, J.J.; Seco, J. KiReS: A Kinect-Based Telerehabilitation System. In Proceedings of the 2013 IEEE 15th International Conference on e-Health Networking, Applications and Services (Healthcom 2013), Lisbon, Portugal, 9-12 October 2013; pp. 444-448. [CrossRef]

17. Yu, X.; Xiong, S. A Dynamic Time Warping Based Algorithm to Evaluate Kinect-Enabled Home-Based Physical Rehabilitation Exercises for Older People. Sensors 2019, 19, 2882. [CrossRef] [PubMed]

18. Chiang, A.-T.; Chen, Q.; Wang, Y.; Fu, M.R. Kinect-Based In-Home Exercise System for Lymphatic Health and Lymphedema Intervention. IEEE J. Transl. Eng. Heal. Med. 2018, 6, 1-13. [CrossRef] [PubMed] 
19. Lin, T.-Y.; Hsieh, C.-H.; Lee, J.-D. A Kinect-Based System for Physical Rehabilitation: Utilizing Tai Chi Exercises to Improve Movement Disorders in Patients with Balance Ability. In Proceedings of the 2013 7th Asia Modelling Symposium, Hong Kong, China, 23-25 July 2013; pp. 149-153. [CrossRef]

20. Su, C.-J.; Chiang, C.-Y.; Huang, J.-Y. Kinect-enabled home-based rehabilitation system using Dynamic Time Warping and fuzzy logic. Appl. Soft Comput. 2014, 22, 652-666. [CrossRef]

21. The Chien Hoang; Ha Trang Dang; Viet Dung Nguyen. Kinect-Based Virtual Training System for Rehabilitation. In Proceedings of the 2017 International Conference on System Science and Engineering (ICSSE), Ho Chi Minh City, Vietnam, 21-23 July 2017; pp. 53-56. [CrossRef]

22. Shapi'i, A.; Bahari, N.N.; Arshad, H.; Zin, N.A.M.; Mahayuddin, Z.R. Rehabilitation Exercise Game Model for Post-Stroke Using Microsoft Kinect Camera. In Proceedings of the 2015 2nd International Conference on Biomedical Engineering (ICoBE), Penang, Malaysia, 30-31 March 2015. [CrossRef]

23. Shapi'i, A.; Arshad, H.; Baharuddin, M.S.; Mohd Sarim, H. Serious Games for Post-Stroke Rehabilitation Using Microsoft Kinect. Int. J. Adv. Sci. Eng. Inf. Technol. 2018, 8, 1654-1661. [CrossRef]

24. Estepa, A.; Sponton Piriz, S.; Albornoz, E.; Martínez, C. Development of a Kinect-based exergaming system for motor rehabilitation in neurological disorders. J. Physics: Conf. Ser. 2016, 705, 012060. [CrossRef]

25. Adolf, J.; Dolezal, J.; Kutilek, P.; Hourova, M.; Hejda, J.; Milerska, I.; Lhotska, L. Automatic Telerehabilitation System in a Home Environment Using Computer Vision. Stud. Health Technol. Inform. 2020, 473, 142-148. [CrossRef]

26. Hassan, H.A.; Abdallah, B.H.; Abdallah, A.A.; Abdel-Aal, R.O.; Numan, R.R.; Darwish, A.K.; El-Behaidy, W.H. Automatic Feedback For Physiotherapy Exercises Based On PoseNet. FCAI-Inform. Bull. 2020, 2, 10-14. [CrossRef]

27. Papandreou, G.; Zhu, T.; Chen, L.-C.; Gidaris, S.; Tompson, J.; Murphy, K. PersonLab: Person Pose Estimation and Instance Segmentation with a Bottom-Up, Part-Based, Geometric Embedding Model. Comput. Vis. ECCV 2018 2018, 282-299. [CrossRef]

28. Papandreou, G.; Zhu, T.; Kanazawa, N.; Toshev, A.; Tompson, J.; Bregler, C.; Murphy, K. Towards Accurate Multi-Person Pose Estimation in the Wild. In Proceedings of the 2017 IEEE Conference on Computer Vision and Pattern Recognition (CVPR), Honolulu, HI, USA, 21-26 July 2017; pp. 4903-4911. [CrossRef]

29. Klimberg, R.K.; Sillup, G.P.; Boyle, K.J.; Tavva, V. Forecasting performance measures - what are their practical meaning? Adv. Bus. Manag. Forecast. 2010, 7, 137-147. [CrossRef]

30. Uddin, S.M.; Rahman, A.; Ansari, E.U. COMPARISON OF SOME STATISTICAL FORECASTING TECHNIQUES WITH GMDH PREDICTOR: A CASE STUDY. J. Mech. Eng. 2018, 47, 16-21. [CrossRef]

31. Dengen, N.; Haviluddin; Andriyani, L.; Wati, M.; Budiman, E.; Alameka, F. Medicine Stock Forecasting Using Least Square Method. In Proceedings of the 2018 2nd East Indonesia Conference on Computer and Information Technology (EIConCIT), Makassar, Indonesia, 6-7 November 2018. [CrossRef]

32. Mandery, C.; Terlemez, O.; Do, M.; Vahrenkamp, N.; Asfour, T. The KIT Whole-Body Human Motion Database. In Proceedings of the 2015 International Conference on Advanced Robotics (ICAR), Istanbul, Turkey, 27-31 July 2015; pp. 329-336. [CrossRef] 